\title{
DIVERSIDAD DE LOS COMPUESTOS ORGÁNICOS BIOACTIVOS DE ORIGEN NATURAL: UNA SINGULARIDAD MANIFESTADA POR LA PLASTICIDAD EN EL METABOLISMO SECUNDARIO
}

Fecha de recepción: 12 de enero de 2016 • Fecha de Evaluación: 14 de abril de 2016 • Fecha de aceptación: 20 de junio de 2016 • Disponible en línea: 25 de julio de 2016

\section{DIVERSITY OF BIOACTIVE NATURALLY-OCCURRING ORGANIC COMPOUNDS: A SINGULARITY EXPRESSED BY THE SECONDARY METABOLISM PLASTICITY}

Kátherin Suárez-Medina1, Ericsson Coy-Barrera ${ }^{1, *}$

\section{RESUMEN}

El uso de compuestos orgánicos de origen natural (i.e., metabolitos secundarios) es una de las formas de aprovechamiento de los organismos más comunes e importantes, entre los que se destacan plantas y microorganismos. La utilidad de estos compuestos se enfoca a diversos escenarios, en el que se resaltan los productos bioactivos, los cuales marcan una fuerte presencia en el control de enfermedades y dolencias. Aunque ya se cuenta con información substancial y significativa relativa a la función que cumplen tales metabolitos secundarios en los organismos que los producen, todavía se desconoce mucho al respecto, sobretodo en el caso de las plantas. Esto se debe quizá a que, en cierta medida, el metabolismo secundario se suele a menudo considerar aparte de las funciones esenciales de la célula. No obstante, existe evidencia de la clara y estrecha relación que se presenta entre metabolismo celular fundamental y las vías bioquímicas que conducen a la síntesis de metabolitos secundarios. Lo anterior permite que el metabolismo secundario se regule por una sofisticada red de comunicación y señalización a escala celular y tisular, lo que le permite manifestarse con el nivel de plasticidad requerido para adaptarse e interactuar con su entorno. Por tanto, debido al hecho que el metabolismo secundario sea tan versátil, permite la biosíntesis de diversos grupos de estructuras biológicamente activas que, por consiguiente, trasciende en la acción y roles de tales productos secundarios, como es el caso de los compuestos fenólicos y las sesquiterpenlactonas.

Palabras Clave: Metabolismo Secundario Vegetal, Bioactivos, Productos Naturales, Sesquiterpenlactonas, Fenólicos.

1 Laboratorio de Química Bioorgánica, Facultad de Ciencias Básicas y Aplicadas, Universidad Militar Nueva Granada. *Autor corresponsal: inquibio@unimilitar.edu.co 


\section{ABSTRACT}

The use of naturally-occurring organic compounds (i.e., secondary metabolites) is one of the most common and important exploitation practices of organisms (predominantly plants and microorganisms). The utility of these compounds is focused on various circumstances, but bioactive products are highlighted, which focus is most related to the control of diseases and disorders. Although there already is substantial and meaningful information regarding the role of such secondary metabolites in those producing organisms, much is still unknown about it, especially in the case of plants. This is perhaps because secondary metabolism is often considered apart from the essential functions of the cell. However, there is evidence of the clear and close relationship that occurs between basic cell metabolism and biochemical pathways leading secondary metabolites synthesis. Secondary metabolism is therefore regulated by a sophisticated communicating and signaling network up to cellular and tissue levels, allowing it to operate at the required level of plasticity to adapt and interact with their environment. Therefore, the fact that secondary metabolism can be too much versatile, it let to the biosynthesis of several groups of biologically active structures thus transcending into the actions and roles of such secondary products, such as phenolic compounds and sesquiterpenlactones.

Keywords: Plant Secondary Metabolism, Bioactives, Natural Products, Sesquiterpenlactones, Phenolics.

\section{INTRODUCCIÓN: METABOLITOS SECUNDARIOS}

Doscientos años de biología y química moderna han descrito la importancia de los metabolitos primarios en las funciones básicas de la vida como la división y crecimiento celular, la respiración, almacenamiento de nutrientes y reproducción. En biología, el concepto de metabolito secundario fue planteado por primera vez por Kossel quien los definió como "opuestos al metabolismo primario" (Kossel, 1891). Treinta años después Czapek aportó al estudio de estos compuestos con la publicación de un libro dedicado a la bioquímica de las plantas el cual nombró "endproduckt". Según él, estos compuestos podían derivarse del metabolismo del nitrógeno por lo que los Ilamó "modificaciones secundarias". En comparación con las moléculas esenciales del metabolismo de las plantas, los metabolitos secundarios pronto serían definidos por su baja abundancia (un poco menos del $1 \%$ del carbono total) (Bourgaud et al., 2001).

A mediados del siglo 20, el desarrollo en las técnicas analíticas permitió el descubrimiento de algunas de las moléculas que componen el metabolismo secundario de las plantas, lo que dio paso al establecimiento de la fitoquímica como disciplina. La cromatografía, una de las técnicas más desarrolladas para este propósito, reveló que algunas de las moléculas de origen natural eran coloreadas debido a la presencia de cromóforos particulares que absorben en la radiación electromagnética de la región del visible; sin embargo, existen otras que son incoloras, así que bajo esta técnica un sinnúmero de moléculas seguirían siendo un misterio. Gracias a estos y otros avances en las técnicas químicas, bioquímicas y el progreso de la 
biología molecular, ha sido posible demostrar que los productos secundarios juegan un papel importante en la adaptación de las plantas al ambiente pues contribuyen al fitness del organismo vegetal a partir de interacciones ecosistémicas, incluso a factores como el cambio climático (Anderson, 2016).

La estructura y patrón con la que se presentan los metabolitos secundarios en las plantas superiores varía de un individuo a otro debido a su complejidad. De hecho se han determinado más de 50.000 estructuras a través de análisis por Resonancia Magnética Nuclear (RMN), Espectrometría de Masas (EM),
Espectroscopía Infrarroja (IR) y difracción de rayos $X$ (DRX) (Seigler, 2003), estimándose que más de un 20\% de las plantas se encuentran sin ser estudiadas por lo que es probable que las estructuras de los metabolitos secundarios superen las 100.000 estructuras. En la tabla 1 se puede apreciar un resumen de las clases y número de estructuras de metabolitos secundarios conocidos (Wink, 2006).

Mientras los metabolitos primarios se encuentran presentes en todas las plantas, los metabolitos secundarios se presentan de manera dispersa y varían entre especies y unidades sistemáticas. Cambian en un

Tabla 1. Núcleos estructurales de los metabolitos secundarios y estructuras conocidas. Modificada de Wink, 2006.

\begin{tabular}{|c|c|}
\hline Clase & Número de estructuras \\
\hline \multicolumn{2}{|c|}{ Con nitrógeno } \\
\hline Alcaloides & 29000 \\
\hline Amino ácidos no proteicos & 700 \\
\hline Aminas & 100 \\
\hline Glicósidos cianogénicos & 60 \\
\hline Glucosinolatos & 100 \\
\hline Alcamidas & 150 \\
\hline Lectinas, péptidos & 800 \\
\hline \multicolumn{2}{|c|}{ Sin nitrógeno } \\
\hline Monoterpenos (incluye iridoides) & 2500 \\
\hline Sesquiterpenos & 5000 \\
\hline Diterpenos & 2500 \\
\hline Triterpenos, esteroides, saponinas. & 5000 \\
\hline Tetraterpenos & 500 \\
\hline Fenilpropanoides, cumarinas, lignanos & 2000 \\
\hline Flavonoides, taninos. & 4000 \\
\hline Policatelinas, ácidos grasos, ceras. & 1500 \\
\hline Poliquetidos (Antraquinonas) & 750 \\
\hline Carbohidratos & 200 \\
\hline
\end{tabular}


órgano o tejido específico principalmente en los diferentes estados de desarrollo entre individuos y poblaciones de plantas (Vialart et al., 2012). No son esenciales para la vida ni para generar energía metabólica vital, pero pueden ser precursores de moléculas esenciales para procesos primarios de las plantas como el caso de las giberelinas. Los metabolitos secundarios también son también considerados como esenciales en la ecología y supervivencia de las plantas (Harborne, 2009).

La clasificación de los metabolitos secundarios se establece a partir de la vía biosintética requerida para su síntesis (por ejemplo, vía del shikimato: compuestos fenólicos, vía acetato - mevalónico: terpenoides), ya que cada uno de ellos es producido a través de una vía metabólica/biogenética que incluye la expresión de enzimas para un sustrato específico (Dewick, 2002; Harborne, 1999b). Los sitios de síntesis pueden diferir entre los tipos de compuestos y especies de plantas, y algunos compuestos pueden ser producidos por todos los tejidos mientras que otros por un único tejido. Esto se debe a que los genes son regulados por un factor de transcripción específico, similar a lo que sucede con los genes que son diferencialmente regulados, así el lugar de síntesis no es necesariamente el sitio de acumulación (Wink, 2006).

En este contexto, los metabolitos también pueden restringirse a expresiones sofisticadas que dependen de la regulación de familias de genes. Así, la vía del shikimato involucra en la síntesis de los lignanos los cuales se encuentran en la mayoría de planta superiores. Sin embargo, existen otros compuestos como los alcaloides, los cuales poseen estructuras altamente diversificadas pero con escasa ocurrencia en las plantas, siendo característicos para grupos taxonómicos particulares. De hecho, la particular distribución de los metabolitos secundarios constituye las bases actuales de la quimiotaxonomía y la química ecológica (Bourgaud, 2001).

El transporte de los compuestos a través de la planta es tan peculiar como su distribución ya que dependiendo de su naturaleza, algunos correrán vía floema y otros vía xilema. Esto es posible mediante la interacción que existe entre los tejidos de conducción para transportar compuestos nitrogenados. Aquellos compuestos hidrofílicos como los alcaloides, aminoácidos, glucosinolatos, glicósidos cianogénicos, flavonoides, taninos, carbohidratos y saponinas son acumulados en las vacuolas, mientras que los metabolitos secundarios lipofílicos como muchos terpenoides son secuestrados en ductos de resina, laticíferos o en células especializadas como las adiposas, tricomas o en la cutícula. Las células epidermales poseen mayor cantidad de metabolitos secundarios ya que son las encargadas de repeler patógenos de la planta y brindarle protección UV (Wink, 2006).

Cabe mencionar que los metabolitos secundarios son el producto final del metabolismo básico de las plantas y es una característica que ellos se almacenen en altas concentraciones en tejidos de reserva, pues le permiten a la planta reproducirse y sobrevivir. Muchos otros metabolitos secundarios no son producto final del metabolismo básico pero pueden ser reciclados. Por ejemplo, el nitrógeno que contiene metabolitos secundarios como los alcaloides, aminoácidos no proteicos o lectinas son acumulados principalmente como compuestos provenientes de nitrógeno tóxico (Rosenthal y Berenbaum, 1992).

\section{Función de los metabolitos secundarios}

Las plantas son organismos que evolutivamente han desarrollado dos tipos de respuestas coordinadas: la mecánica y la química. En esta última intervienen los metabolitos secundarios ya que a partir de un estímulo externo, ya sea por patógenos como hongos, virus, bacterias, por plagas e incluso por el contacto con otra planta, se desencadenan señales químicas en el organismo vegetal que resultan en la síntesis/expresión/liberación de metabolitos secundarios. Esto se debe a que estos compuestos hacen 
parte del mecanismo de defensa de las plantas. En la figura 1 se esquematiza de manera general la función de los metabolitos secundarios (Li et al., 1993; Swain, 2007).

Dado que la biosíntesis de muchos metabolitos secundarios es constitutiva (i.e., metabolitos producidos de manera permanente y dependiente de la activación genética en condiciones normales/sanas y que se constituyen como la primera barrera de interacción/defensa de la planta), las concentraciones y sitio en las que se encuentran en la planta son fundamentales para que estos puedan actuar de manera adecuada frente a un estímulo ambiental o una condición de estrés biológico, por ejemplo, una herida o infección. Estos factores desencadenan una respuesta en la planta que resulta en la activación de genes para expresión de enzimas que favorecerán la síntesis bioquímica de metabolitos secundarios. Así, por ejemplo, la activación de genes puede conducir a la vía biosintética del ácido jasmónico o ácido salicílico, las cuáles se comportan como elicitores en las reacciones de defensa de las plantas (Kessler et al., 2004), siendo considerados como fitohormonas con múltiples funciones. Los jasmonatos (i.e., compuestos biosintéticamente relacionados al ácido jasmónico y al jasmonato de metilo) juegan roles trascendentales tanto en la señalización a estímulos y estrés biótico/abiótico como en procesos relacionados con crecimiento y desarrollo vegetal (Avanci et al., 2010). Otros metabolitos son almacenados como pro-sustancias bioactivas que se liberan como estrategia de adaptación. Un ejemplo conocido es la activación de hidrolasas que favorecen la ruptura de glicósidos, previamente almacenados/acumulados en las vacuolas, para liberar la correspondiente aglicona biológicamente activa que cumplirá la función adaptativa (Tabla 2).

Por otro lado, los estímulos ambientales o condiciones de estrés biológico también pueden desencadenar la síntesis de metabolitos secundarios inducibles (i.e., aquellos compuestos que no están

\begin{tabular}{|c|c|c|c|c|c|c|}
\hline \multirow{5}{*}{$\begin{array}{l}\text { Función de los } \\
\text { Metabolitos } \\
\text { Secundarios }\end{array}$} & \multicolumn{6}{|c|}{$\longrightarrow$ - Protección UV } \\
\hline & $\longrightarrow$ & Atracción & $\rightarrow$ & \multicolumn{3}{|c|}{$\begin{array}{l}\text { - Insectos polinizadores } \\
\text { - Animales dispersores de semillas } \\
\text { - Herbívoros especialistas }\end{array}$} \\
\hline & & & $\longrightarrow$ & $\begin{array}{l}\text { - Competencia entre } \\
\text { plantas }\end{array}$ & $\longrightarrow$ & $\begin{array}{l}\text { - Inhibición de: germinación } \\
\text { y crecimiento de plántulas }\end{array}$ \\
\hline & $\longrightarrow$ & Defensa & $\longrightarrow$ & $\begin{array}{l}\text { - Microbios/virus: } \\
\text { - Hongos } \\
\text { - Bacterias }\end{array}$ & & $\begin{array}{l}\text { - Toxicidad } \\
\text { - Inhibición de crecimiento }\end{array}$ \\
\hline & & & $\rightarrow$ & $\begin{array}{l}\text { - Herbívoros: } \\
\text { - Insectos } \\
\text { - Moluscos } \\
\text { - Vertebrados }\end{array}$ & $\longrightarrow$ & $\begin{array}{l}\text { - Repelencia } \\
\text { - Disuasión } \\
\text { - Toxicidad } \\
\text { - Inhibición de crecimiento }\end{array}$ \\
\hline
\end{tabular}

Figura 1. Función de los metabolitos secundarios. Modificada de Wink, 2006. 
Tabla 2. Típicas pro-sustancias bioactivas activadas por heridas o infecciones en las plantas. Modificada de Wink, 2006.

\begin{tabular}{cc}
\hline Metabolito acumulado & Aleloquímico bioactivo \\
\hline Glicósido cianogénico & HCN \\
\hline Glucosinolato & Isotiocianato \\
\hline Aliína & Alicina \\
\hline Coumaroilglicósido & Cumarina \\
\hline Arbutina & Quinona \\
\hline Salicina, salicilato de metilo & Sulidenina, ácido salićlico \\
\hline Geina & Saponina Mono-desmosídica \\
\hline Saponinas Bi-desmosídicas & Metilazoximetanol (NAM) \\
\hline Cicasina & Protoanemonina
\end{tabular}

presentes en plantas en condiciones normales/sanas y se producen a razón del estímulo externo, debido a la expresión de señales químicas que se generan de forma local o sistémica), siendo las fitoalexinas un ejemplo reconocido de tal evento. Este grupo de compuestos se caracteriza por tener bajo peso molecular y poseer actividad antimicrobiana. Un ejemplo del papel que juegan las fitoalexinas en las plantas es el capsidiol (Figura 2), correspondiente a un compuesto que tiene como precursor el aristoloqueno, y que se sintetiza en las hojas de tabaco. Específicamente es un sesquiterpeno bicíclico dihidroxilado el cual se produce en un gran número de solanáceas como respuesta a un estímulo ambiental. En Nicotiana tabacum, es el primer compuesto antimicrobiano que se produce como respuesta a la activación de genes específicos por presencia de un fitopatógeno (Ralston et al., 2001). Curiosamente, se ha demostrado que la vía metabólica en la que se sintetiza el capsidiol es influenciada por Manduca sexta (polilla americana) y que el punto metabólico crucial para la síntesis del compuesto es la formación de una unidad bicíclica, la cual se forma a partir de isoformas de terpenosintasas [e.g., 5-epi-aristoloquensintasa (EAS)] encargadas de ciclar la unidad de un prenil fosfato para dar forma a esqueletos de carbono de aristoloqueno (Bohlmann et al., 2002). Una vez formados los esqueletos de carbono se da inicio a la síntesis del capsidiol (Maldonado-Bonilla et al., 2008).

Además de estar relacionados en procesos de defensa de la planta, los metabolitos secundarios se comportan como compuestos de señalización al cumplir un papel crucial en procesos de polinización y dispersión de semillas mediante la atracción de insectos a la planta. Ejemplo de ello son los compuestos volátiles presentes en aceites esenciales y los flavonoides con color (e.g., antocianidinas), los cuales generan en las plantas aromas y colores, respectivamente, atractivos para insectos polinizadores y dispersores (Hadacek, 2002).

Como complemento estratégico a la defensa química, un gran número de plantas utilizan rasgos mecánicos y/o morfológicos como protección dentro de las cuales se pueden citar espinas, pelos glandulares y punzantes, los cuales, por lo general, están llenos de compuestos químicos tóxicos/nocivos. En las plantas perenes leñosas es usual encontrar grandes espinas (algunas de ellas venenosas) que funcionan como trampa contra enemigos (van Wyk, 2004). 
Influencias exógenas en los niveles de los metabolitos secundarios de las plantas

Los aspectos mencionados anteriormente, incluyendo aquellos en los que se involucra una interacción planta-planta, pueden originarse por efectos bióticos y abióticos, los cuales son los responsables de la respuesta adquirida en las plantas. Son causantes de cambios en la cantidad de metabolitos secundarios a sintetizar y/o acumular en la planta según el estímulo que el organismo vegetal perciba (Wink, 2003).

Los efectos abióticos incluyen todos aquellos efectos físicos que gobiernan un hábitat como lo son la luz, radiación ultravioleta, disponibilidad de agua (incluye la sequía), temperatura y composición del suelo. Algunos de los metabolitos secundarios, definidos químicamente como compuestos fenólicos debido a su variada estructura de esqueletos de carbono, median su biosíntesis y bioactividad a través de la intensidad de la luz UV. Un claro ejemplo a esto son las cumarinas. Estos metabolitos son sintetizados y acumulados en los tejidos de las plantas, principalmente en las hojas, en forma no tóxica a través de un ciclo estacionario. Dicho proceso es mediado por genes inducidos por la acción UV, el cual una vez activo, desencadena la señalización para determinar los niveles no tóxicos de cumarina que deben acumularse en los tejidos de la planta según su estado fenológico para su protección (Ashihara et al., 2008)

La luz tiene también efectos importantes en la pre-cosecha de algunas especies vegetales como en los brotes de Camellia sinensis (el conocido té verde). La síntesis de derivados fenólicos de tipo flavonoles y
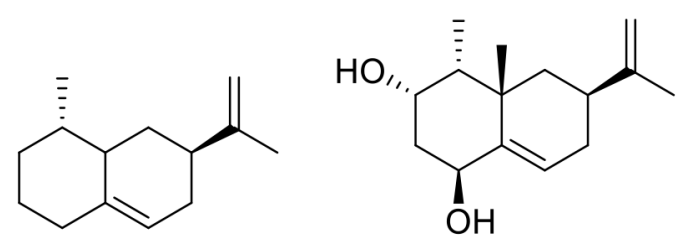

Figura 2. Estructura química del aristoloqueno y el capsidiol. catequinas es regulada mediante la sobreexposición de los brotes a la luz, lo que genera la acumulación de compuestos fenólicos en las hojas de la planta. De esta manera, la exclusión de los brotes a la sobreexposición de luz antes de la cosecha permite aumentar los niveles de alcaloides tipo purinas, como la cafeína, permitiendo aumentar la calidad de la cosecha (Wang et al., 2011).

Estudios fitoquímicos han demostrado que tanto la salinidad como la sequía resultan en la sobreproducción de especies reactivas de oxígeno (ROS), lo cual interrumpe las funciones celulares debido al estrés oxidativo al que es sometido la célula (Demidchik, 2015; Foyer \& Shigeoka, 2011; Phung et al., 2011). En Arabidopsis, la acumulación de ciertas moléculas generan la sobreproducción de ROS que participan en la inducción, señalización y ejecución de la muerte celular (van Breussegmen \& Dat, 2006). Asimismo, la deficiencia de ácido ascórbico (AsA) origina alteraciones en el crecimiento de la planta (Kerchev et al., 2011) y mejora la tolerancia cuando se recibe de forma exógena (Azzedine et al., 2011).

La presencia de antioxidantes en las plantas, tales como el glutatión, ascorbato y poderosos antioxidantes como los polifenoles/flavonoides, es altamente esencial, ya que permiten regular las alteraciones oxidativas en el metabolismo, equilibran las cantidad de ROS en la célula, sitio de producción de ROS y el sistema funcional de los antioxidantes (la planta recupera tolerancia al estrés abiótico) (Gechev et al., 2006).

En células guardianas, la señalización celular y cierre de estomas son regulados a través del balance que debe existir entre prooxidantes (radicales libres), como el $\mathrm{H}_{2} \mathrm{O}_{2}$ y el antioxidante AsA, con lo cual se asegura el adecuado estado redox del AsA y niveles de prooxidantes (Li et al., 2009). Además de jugar un papel importante en estos procesos, el AsA es necesario como regulador biológico en las plantas así como el ácido abscísico y el etileno. Cabe citar que son fundamentales en el florecimiento y senescencia de las mismas (Chen \& Gallie, 2008). 
Asimismo, los factores bióticos incluyen todas las interacciones entre la bioquímica de la planta y su fisiología. De una manera más amplia podría asumirse que los efectos bióticos están relacionados tanto con la interacción de la planta con los microorganismos como con sus aspectos fisiológicos, i.e., fenología y ontogenia (Briskin, 2000).

La fenología de la planta hace referencia a la manera en que la fisiología de la planta opera a través del tiempo, más específicamente se entiende como la distribución de las fenofases de la planta a lo largo de las estaciones climáticas (o aquellas condiciones climáticas en países intertropicales) lo cual puede explicar la variabilidad del metabolismo de las plantas durante las estaciones. En otras palabras, la fenología describe cómo las variables meteorológicas afectan a las manifestaciones periódicas o estacionales de las plantas, e.g., todos los cambios que se producen durante los ciclos ultradianos, circadianos y estacionales de un organismo vivo, como el florecimiento en las angiospermas o la senescencia de las gimnospermas en climas templados (Minto \& Blacklock, 2008). Por su parte, la ontogenia determina el desarrollo de un organismo desde el óvulo hasta su senescencia. Dentro de todo este proceso hay cambios fisiológicos permanentes que pueden ser desencadenados por interacciones con el medio ambiente o por su misma dinámica. Ejemplo a esto son los genes asociados a la síntesis de derivados de ácidos grasos como el poliacetileno durante la floración de Helianthus annus. Estos genes se sobreexpresan específicamente en dicho estado fenológico que en cualquier otro estado de la planta. Se ha comprobado que existe una mayor expresión en las hojas que en las semillas en un incremento de $10^{4}$ veces (Minto \& Blacklock, 2008).

La fisiología de las plantas también se ve afectada por presencia de microorganismos como patógenos. Como se ha mencionado, los metabolitos secundarios son sintetizados por vías metabólicas que son activadas por una cascada de señalización que se produce en el instante que la planta detecta la presencia del patógeno. En algunos casos, la acumulación y actividad biológica de los metabolitos secundarios es limitada por factores químicos, físicos y biológicos que generan su degradación (Kaur, et al., 2009). Caso específico se evidencia en aquellos metabolitos secundarios que son liberados al suelo por la planta. La degradación que éstos sufren en el suelo genera un cambio estructural o una modificación en la dinámica de los metabolitos secundarios, resultando en una alteración de su actividad (Inderjit \& Weiner, 2001; Perrry et al., 2007). Un ejemplo a citar es la bacteria Acinetobacter calcoaceticus, la cual puede convertir la 2(3H)-benzoxazozina (BOA) en 2,2'oxo-1,1'-azobenceno (AZOB). Este último es más inhibitorio en algunas plantas (Jilani et al., 2008). Los compuestos fenólicos de algunos líquenes tienen un efecto antibiótico in vitro sobre algunos organismos del suelo pero son mucho menos activos en condiciones naturales (Stark et al., 2007). Estudios han demostrado que las plantas invasoras como Centaurea maculosa pueden afectar el crecimiento y germinación de plantas nativas al exudar desde sus raíces la fitotoxina (-)-catequina. En especies susceptibles, como Arabidopsis thaliana, el efecto alelopático de estas especies invasoras, desencadena una respuesta ROS en la planta nativa. Inicia en el meristemo de la raíz, desencadena una cascada de señalización de $\mathrm{Ca}^{2+}$, genera cambios en la expresión de los genes del genoma total y finaliza con la muerte del sistema radicular (Bais, et al., 2003).

\section{Bioactividad de los metabolitos secundarios}

La bioactividad de los metabolitos secundarios empezó a ser estudiada a través de experimentación in vivo en plantas venenosas. Aún en la actualidad, la industria farmacéutica aprovecha los beneficios que éstas le ofrecen al hombre, razón por la que es uno de los principales sectores en explotación vegetal. Sin embargo, a pesar de los grandes proyectos de 
protección y conservación de la biodiversidad mundial, los hábitats que albergan plantas que acumulan metabolitos secundarios bioactivos aún son desentendidos de estudios, lo cual pone en riesgo la supervivencia y aprovechamiento de estas especies (Aguilar-Støen \& Moe, 2007).

Los compuestos bioactivos son constituyentes que se producen naturalmente en pequeñas cantidades en las plantas y frutos (Kris-Etherton et al., 2002). Muchos de los compuestos bioactivos incluyen metabolitos secundarios estructuralmente variados entre los que se cuentan las micotoxinas, alcaloides, factores de crecimiento en las planta, compuestos fenólicos, lignanos, salicilatos, estanoles, esteroles, glicosinolatos, entre otros (Hooper \& Cassidy, 2006; Nigam, 2009). Dentro del grupo de los compuestos fenólicos mencionados anteriormente, se incluyen los flavonoides, ácidos fenólicos, taninos, entre otros. De hecho, las plantas poseen tan alto contenido de compuestos fenólicos que son consideradas excelente fuente para aplicaciones terapéuticas, nutricionales y fitoquímicas debido a las propiedades alelopáticas, antifúngicas, bactericidas, entre otras, que poseen (Pandey \& Rizvi, 2009).

Otra clase importante de compuestos fenólicos son los ácidos fenólicos; se caracterizan por poseer funciones bioactivas y encontrarse en un gran número de plantas y alimentos. Pueden dividirse en dos subgrupos de acuerdo a su estructura: ácidos hidroxibenzóicos e hidroxicinnámicos (Figura 3). Los ácidos hidroxibenzóicos más comunes incluyen el ácido gálico, ácido p-hidroxibenzóico, ácido protocatecuico, vainillina y ácido siríngico. Por su parte, los ácidos hidroxicinnámicos más comunes son el caféico, ferúlico, p-cumárico y sinápico (Conforti et al., 2009). Respecto al grupo de los flavonoides, el mayor número de flavonoides, i.e., flavonoles, flavonas, flavononas, flavonoles, isoflavonas y antocianinas, resultan de variaciones estructurales que se producen por sustituciones selectivas en sus anillos. La Figura 4 muestra ejemplos de los flavonoides que se presentan con mayor frecuencia en la naturaleza, siendo los flavonoides los compuestos que constituyen el grupo más grande de los fenoles (Harborne et al., 1999b; Martins et al., 2011). Muchos de estos compuestos fenólicos son capaces de producir disrupción en la pared celular de hongos patógenos. Dicha pared está compuesta estructuralmente por una matriz de proteínas y policarbohidratos que, debido a un daño estructural en ella, puede ocasionar el mal funcionamiento de la pared celular haciendo a las células del hongo sensibles a lisis osmótica y fungicidas (Baya et al., 2001).

En este contexto, los compuestos bioactivos han sido de gran interés debido a que son sintetizados por vías metabólicas complejas y regulados de maneras sofisticadas (Yazaki, 2004). Por tanto, el poder comprenderlas representa una oportunidad grande para el aprovechamiento de los beneficios que pueden ofrecer para la salud humana, animal y vegetal. Dentro de los mecanismos de acción que han sido demostrados se describen la capacidad de reducir la incidencia de ciertas enfermedades severas ocasionadas por hongos y bacterias, reducir el crecimiento del tubo germinativo en hongos, reducir la germinación de esporas de bacterias y hongos, inhibir la síntesis de proteínas, modular la estructura de microtúbulos, entre muchos otros efectos (Jiménez et al., 2008).

Adicionalmente, debido a los beneficios que puede proporcionar la bioactividad de estos compuestos en el manejo integrado de plagas en cultivos económicamente importantes y salud humana, los estudios enfocados a la búsqueda de vegetales, frutas, plantas, residuos agrícolas y agroindustriales que sean fuente de compuestos fenólicos se han intensificado permanentemente (Kircher \& Lieberman, 1967; Ogita, 2003).

Los compuestos bioactivos se encuentran en ciertos órganos de la planta. Este evento se debe a que los genes encargados de la biosíntesis de dichos compuestos suelen expresarse en mayor proporción en órganos específicos, por lo general donde los metabolitos se 
acumulan. No obstante, a menudo el órgano dónde se acumulan estos compuestos es diferente al de biosíntesis. Por tanto, en estos casos, los metabolitos secundarios son translocados a otra parte de la planta, tal y como sucede con la nicotina. Los genes de este alcaloide producido por Nicotiana spp. se expresan normalmente en los tejidos de las raíces (órganos fuente) pero son transportados a la parte aérea de la planta para ser acumulados en las hojas (órgano sumidero). Esto quiere decir que cada metabolito tiene un órgano sumidero al cual pertenece distribuyéndose de manera heterogénea en la planta sin importar el tejido en el que los genes se expresen. Es por este motivo que al evaluar la bioactividad de los metabolitos secundarios de cierta especie es necesario obtener extractos de diferentes órganos de la planta (Shoji, 2000).
Como ejemplo de órgano sumidero de compuestos bioactivos, se puede citar el tronco de las plantas, principalmente la madera. En esta estructura los compuestos acumulados son principalmente antifúngicos. En los extractos etanólicos de madera de Calocedru macrolepis y Cupressus lusitánica, se ha reportado la presencia de dos fitoalexinas importantes en las respuestas antifúngicas de las plantas, $\beta$-thujaplicina y $\gamma$-thujaplicina. Su mecanismo se atribuye principalmente a los cambios que generan en la permeabilidad de la pared celular. Una vez el compuesto interactúa con las células del fitopatógeno, el citoplasma celular se rompe y los metales son quelados, lo que finalmente lleva a la interrupción de los procesos enzimáticos del hongo (Yen, et al., 2008).

En las hojas, específicamente en la cera epicuticular, se encuentran compuestos homólogos a los

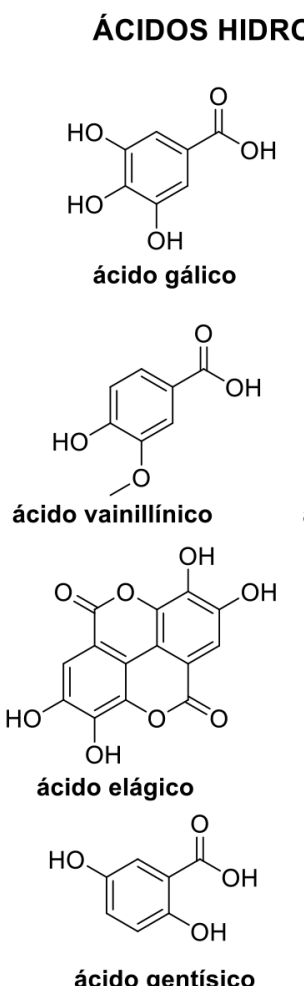<smiles>CC(C)(C)COc1cc(C(=O)O)ccc1O</smiles><smiles>COc1cc(/C=C/C(=O)O)ccc1O</smiles><smiles>O=C(O)/C=C/c1ccc(O)c(O)c1</smiles>

ácido ferúlico<smiles>O=C(O)c1ccc(O)cc1</smiles>

ácido p-hidroxibenzóico<smiles>O=C(O)/C=C/c1ccc(O)cc1</smiles><smiles>COc1cc(/C=C/C(=O)O)cc(OC)c1O</smiles>

ácido p-cumárico<smiles>COc1cc(C(=O)O)cc(OC)c1O</smiles>
ácido siríngico<smiles>O=C(O)c1ccccc1O</smiles>
ácido salicílico<smiles>O=C(O)/C=C/c1ccccc1</smiles>

ácido cinámico

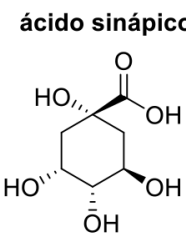

ácido quínico<smiles>O=C(/C=C/c1ccc(O)c(O)c1)OC1C(O)C2CC(O)C(O)C1C2O</smiles>

ácido clorogénico

Figura 3. Ejemplo de ácidos fenólicos que se producen naturalmente. Modificada de Martins et al., 2011 
hidrocarburos como ésteres, acetonas, alcoholes, terpenoides y ácidos carboxílicos. Los flavonoides también se encuentran presentes y son los responsables de otorgarle a la planta cierta actividad antifúngica a pesar de estar asociados principalmente a procesos de protección UV (Barker, 1982). Se ha demostrado que los flavonoides son capaces de inhibir el crecimiento de cepas de Fusarium oxysporum (Galeotti et al., 2008). Los metabolitos secundarios no son solo activos contra hongos, pueden generar efecto sobre bacterias como aquellos presentes en Cinnamommum zeylanicum, los cuales son capaces de generar efecto inhibitorio en el crecimiento de Escherichia coli desde concentraciones mínimas de $64 \mu \mathrm{g} / \mathrm{mL}$ (Voukeng et al.2012).

Los metabolitos secundarios incluso son producidos/almacenados en organelos específicos dentro de la célula, en un suceso conocido como compartimentación (Roze et al., 2011). Esta situación marca una clara conexión entre el metabolismo celular fundamental y las vías bioquímicas que conducen a la síntesis de metabolitos secundarios, dado que, sumado a la utilización de aquellos precursores bioquímicos cruciales que son compartidos con los procesos más esenciales de la célula (e.g., aminoácidos, acetilCoA, NADPH, ATP), aquellas enzimas que catalizan la producción de metabolitos secundarios están claramente compartimentadas en sitios subcelulares altamente conservados que condicionan su posición con el fin de utilizar estos precursores bioquímicos comunes (Kistler \& Broz, 2015).

Dentro de la plétora de compuestos con bioactividad derivados del metabolismo secundario vegetal, es necesario mencionar a los sesquiterpenos. Estos constituyen, dentro de las plantas, un grupo diverso de compuestos químicos con variada actividad biológica; actúan como agentes alelopáticos, citotoxicos, antitumorales y antifúngicos. Han sido descritos en las familias Acanthaceae, Anacardiaceae, Apiaceae, Euphorbiaceae, Lauraceae, Magnoliaceae, Menispermaceae, Rutaceae, Winteraceae y Hepatidae. Sin embargo, el mayor número de sesquiterpenos se encuentra en la familia Compositae (Asteraceae) con más de 3000 estructuras diferentes reportadas (Modzelewska et al., 2005).

Estos compuestos son una clase de terpenoides que ocurren de manera espontánea en las plantas. Representan una clase única y diversa dentro de los productos naturales y dentro de los aceites esenciales. Estos se forman por la condensación de tres unidades de isopreno y ciclaciones subsecuentes y transformaciones oxidativas para producir fusiones cis y trans. Entre los sesquiterpenos reconocidos como más activos e importantes se resaltan las sesquiterpenlactonas (SLs), cuyo rasgo característico es la presencia de un anillo $\gamma$-lactona caracterizado por poseer un grupo a-metileno (Picman, 1986). Es también común la incorporación de hidroxilos, metoxilos, hidroxilos esterificados y epóxidos. Pocas SLs ocurren en forma de glicósido y algunos contienen átomos de halógeno o azufre (Chen, 1995). Las SLs son clasificadas de acuerdo al esqueleto carboxílico presente en los pseudoguaninólidos, guaianólidos. germanocranólidos, eudesmanólidos, heliangólidos y hiptocretólidos (Figura 5)

La mayoría de las SLs han mostrado poseer actividad citotóxica y actividad in vivo contra leukemia P388. Estudios en los que se relaciona la estructura con la actividad, han demostrado que varias SLs citotoxicas reaccionan con tioles, como los residuos de cisteína. Estas reacciones están mediadas químicamente por un sistema basado en un carbonilo $\alpha$, $\beta$ - insaturado presente en las sesquiterpenlactonas. Estos estudios además de determinar la relación función-estructura de dichos compuestos, soportan el planteamiento que las SLs inhiben el crecimiento de tumores a través de una alquilación selectiva de biomoléculas reguladoras como las enzimas fundamentales. Estas enzimas controlan la división celular $y$, por ende, inhiben una variedad de funciones celulares que dirigen a la célula a la apoptosis. La actividad 

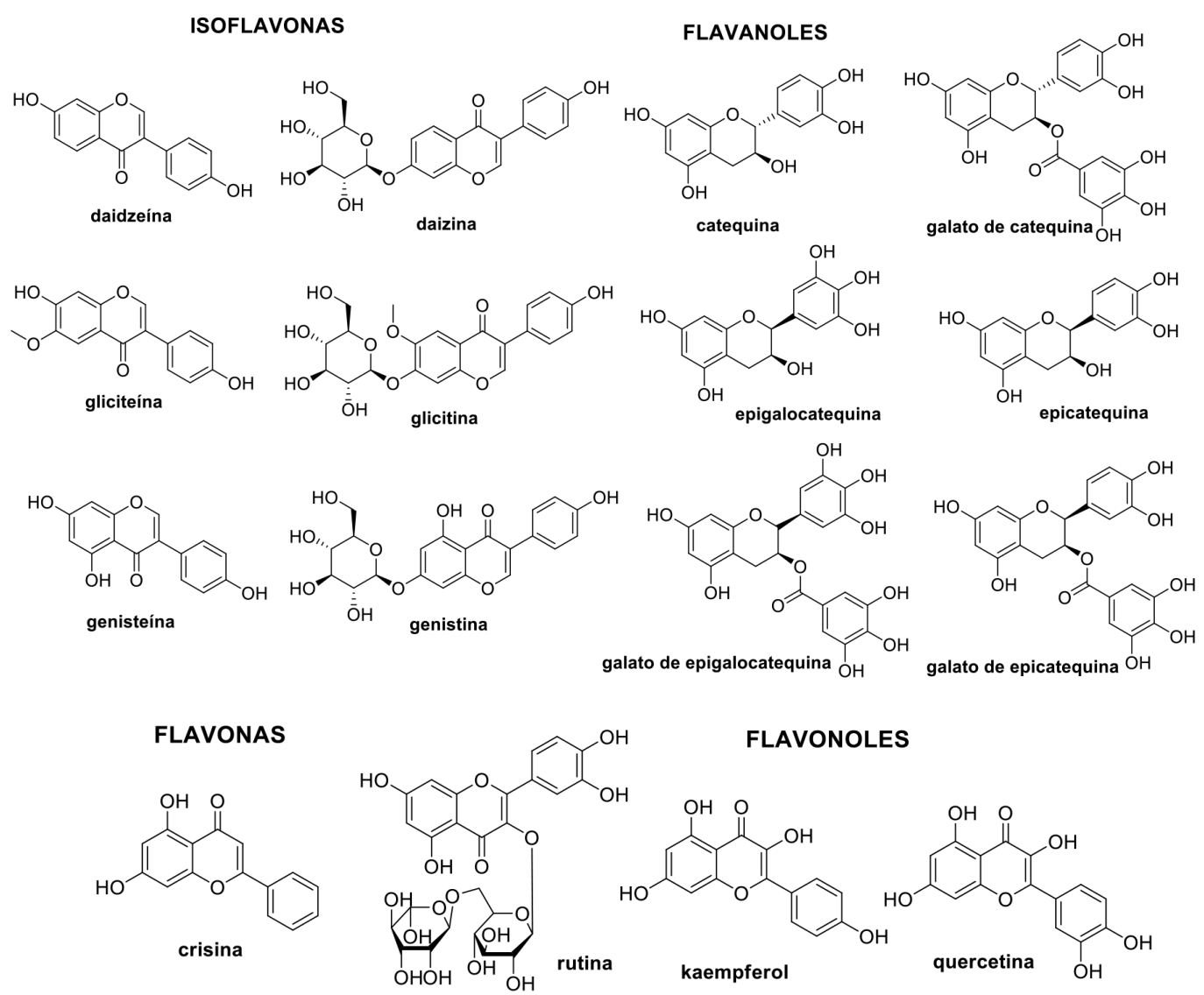

FLAVONOLES<smiles>O=c1cc(-c2ccc(O)cc2)oc2cc(O)cc(O)c12</smiles><smiles>O=c1cc(-c2ccc(O)cc2)oc2cc(O)cc(O)c12</smiles><smiles></smiles>

\section{FLAVANONAS}
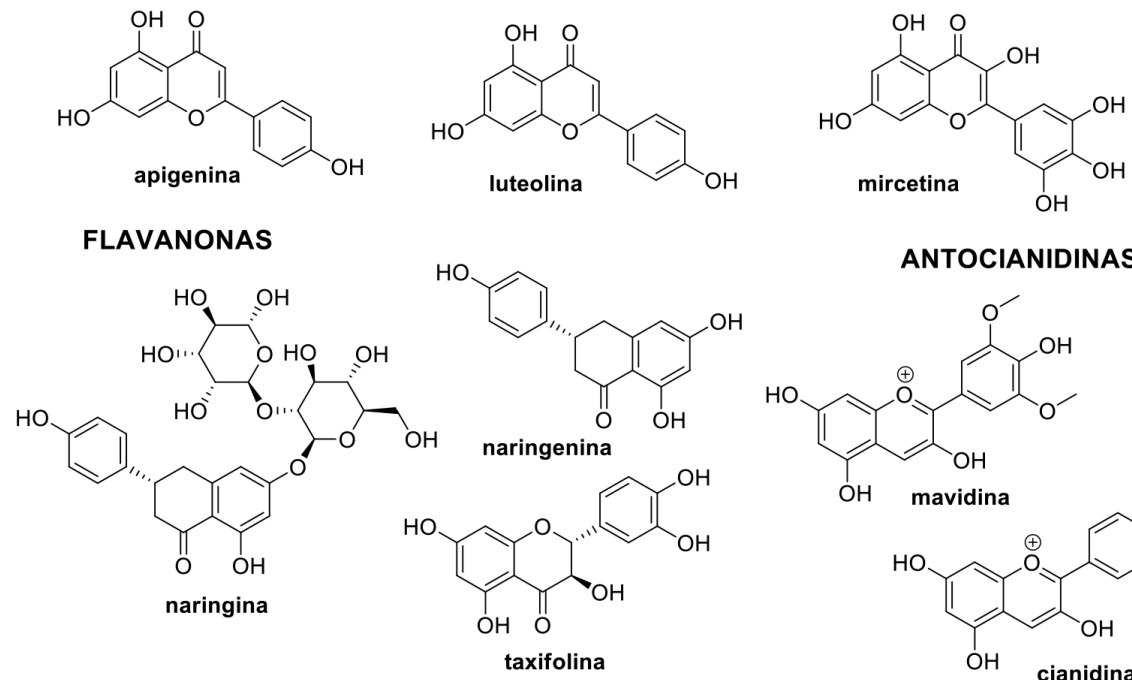

ANTOCIANIDINAS

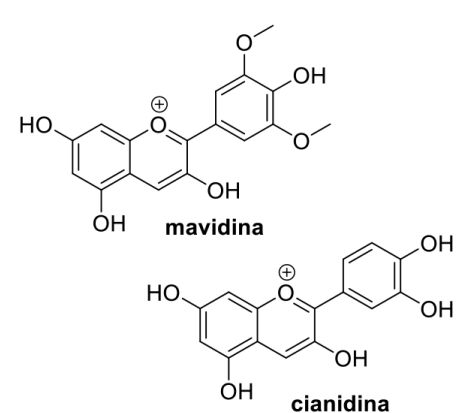

Figura 4. Ejemplo de flavonoides que se producen naturalmente. Modificada de Martins et al., 2011. 
individual de las SLs difiere entre sí posiblemente por los numerosos elementos estructurales alquilantes, sin embargo otros factores como la lipofilicidad, geometría molecular y ambiente químico o el blanco sulfidrilo pueden influenciar en la función de las SLs. En la figura 6 se muestran algunas estructuras que llevan a la diversidad estructural de las SLs (Chaturvedi, 2011).

En este sentido, las SLs se han relacionado a mecanismo de acción muy específicos contra el cáncer (e.g., costunólido, partenólido, helenalina, artemisina y sus derivados), pero también se describen efectos antiinflamatoriaos (costunólido, partenólido, helenalina), antiparasitarios (i.e., antimaláricos) (artemisina y sus derivados), antivirales, antibacteriales y antifúngicas (Picman, 1986).

Gran número de estudios han corroborado la presencia de sesquiterpenos antifúngicos en plantas superiores o sus extractos. Ejemplo a ello es la fuerte inhibición de xantatina en el crecimiento de
Trichophyton mentagrophytes y Candida albicans. La helenalina e isohelenalina inhibieron el crecimiento de 16 especies de hongos en diferentes grados de severidad, presentándose el mayor en Microsporum cookei, Trichophyton mentagrophytes y Trichothecium roseum. Las dos lactonas han sido reportadas previamente contra tres dermofitos, Trichophyton gypseum, T. acuminatum y Epidermophyton sp. (Picman, 1983; Ahmed \& Abdelgaleil, 2005). Algunos otros sesquiterpenos han demostrado ser fungistáticos como la alantolactona contra Fusarium solani. La partenina mostró inhibición de la germinación del esporangio y la motilidad de las zoosporas de Sclerospora graminícola (Pandey, 2009). Asimismo, una serie de SLs aisladas de dos especies del género Centaurea se evaluaron contra nueve especies de hongos, demostrado sensibilidad a a-hidroxi4-epi-soncucarpólido y 40-acetoximalacitanólido (Skaltsa et al., 2000). El primero, al ser comparado
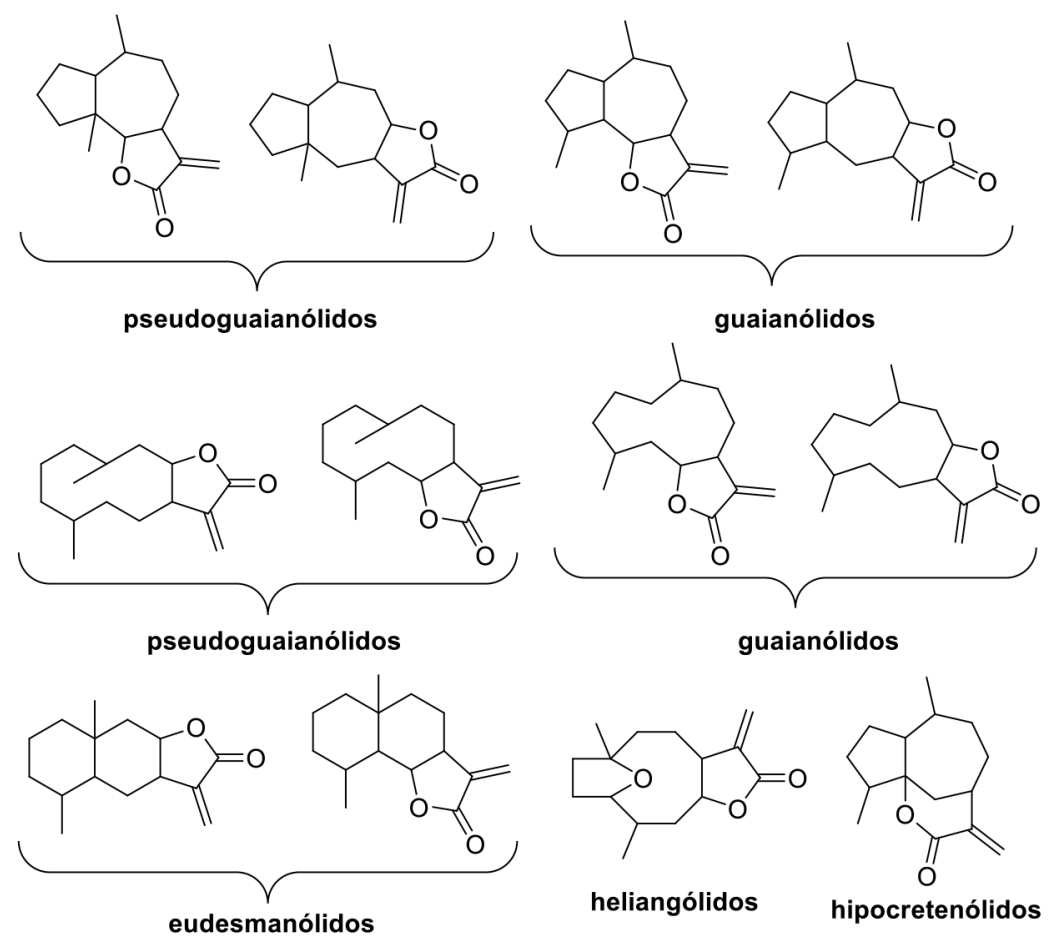

Figura 5. Esqueletos básicos de los sesquiterpenlactonas. Modificada de Chaturvedi, 2011. 


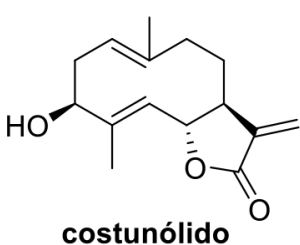<smiles>C=C(C)C(=O)OC1C(OC(=O)C(=C)CO)CC(=C)C2CC(O)C(=C)C21</smiles>

cinaropicrina<smiles>C=C1C(=O)OC2CC(C)C3C=CC(=O)[C@@]3(C)C(O)C12</smiles>

helenalina

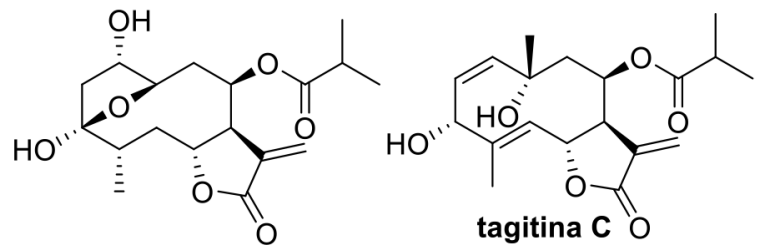

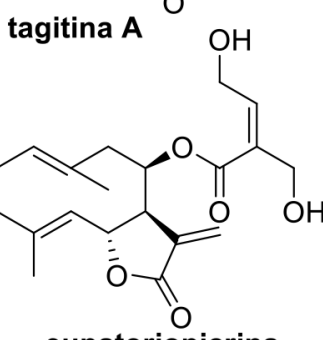

eupatoriopicrina

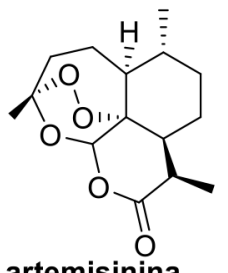

artemisinina

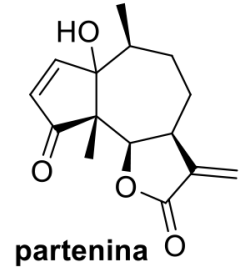<smiles>C=C[C@]12COC(=O)C(=C)[C@H]1[C@@H]1OC(=O)C(C)(C)[C@H]1[C@@H]2OC(=O)C(=C)CO</smiles>

vermodalina

Figura 6. Diversidad estructural de los Sesquiterpenos lactonas. Modificada de Chaturvedi, 2011.

con el 40-acetoximalacitanólido mostró mayor actividad contra todas las especies evaluadas a excepción de Cladosporium cladosporioides.

Bajo este contexto, un gran número de estudios afirman lo planteado anteriormente, cuyos ejemplos se citan en detalle en trabajos previos (Hansson et al., 2012; Meng et al., 2001; Saad et al., 2012; Nuñez et al., 2007) los cuales llevan a indicar que los sesquiterpenos presentan bioactividad contra una amplia variedad de microorganismos. No obstante, aunque se reportan numerosos sesquiterpenos bioactivos, el mecanismo de acción mantiene avances mínimos, siendo en la actualidad un aspecto poco conocido en el estudio de la bioactividad de tales metabolitos contra microorganismos.

En conclusión, el uso de metabolitos secundarios seguirá como una de las formas de aprovechamiento más comunes e importantes de plantas y microorganismos y, a medida que siga avanzando el conocimiento sobre la conexión entre el metabolismo celular y las vías de síntesis de metabolitos secundarios, tal aprovechamiento será cada vez más beneficioso. Todo lo anterior demarcado a partir de aquella plasticidad que las plantas han desarrollado a lo largo del tiempo y que, por ende, requiere cada vez más de estudios conducentes a descifrar/descubrir/entender el rol del metabolismo secundario en las plantas y otros organismos.

\section{AGRADECIMIENTOS}

Los autores agradecen a la Universidad Militar Nueva Granada por la financiación. Producto derivado del proyecto INV-CIAS-2050 financiado por la Vicerrectoría de Investigaciones de la UMNG Vigencia 2015. 


\section{REFERENCIAS}

1 Aguilar-Støen, M., Moe, S.R. 2007. Medicinal plant conservation and management: distribution of wild and cultivated species in eight countries. Biodiversity and Conservation 16: 1973-1981.

2 Ahmed, S., Abdelgaleil, S. 2005. Antifungal activity of extracts and sesquiterpenes from Magnolia grandiflora L. (Magnoliaceae). International Journal of Agriculture and Biology 7: 638-642.

3 Anderson, J.T. 2016. Plant fitness in a rapidly changing world. New Phytologyst 210(1): 81-7.

4 Ashihara, H., Sano, H., Crozier, A. 2008. Caffeine and related purine alkaloids: biosynthesis, catabolism, function and genetic engineering. Phytochemistry 841-856.

5 Avanci, N.C., Luche, D.D., Goldman, G.H., Goldman, M.H. 2010. Jasmonates are phytohormones with multiple functions, including plant defense and reproduction. Genetics and Molecular Research: 484-505.

6 Azzedine, F., Gherroucha, H., Baka, M. 2011. Improvement of salt tolerance in Durum wheat by ascorbic acid application. Physiology and Biochemestry 7: 27-37.

7 Bais, H.P., Vepachedu, R., Gilroy, S., Callaway, R.M., Vivanco, J.M. 2003. Allelopathy and exotic plant invasion: from molecules and genes to species interactions. Science 301: 1377-1380.

8 Baker, E.A. 1982. Chemistry and morphology of plant epicuticular waxes. In: Cutler D.J., Alvin K.L., Price C.E (eds). The Plant Cuticle. Londres, Academic Press, 139-165pp.

9 Baya, M., Soulounganga, P., Gelhaye, E., Gerardin, P. 2001. Fungicidal activity of b-thujaplicin analogues. Pest Manage Science 57: 833- 838.

10 Bohlmann, J., Stauber, E.J., Krock, B., Oldham, N.J., Gershenzon, J., Baldwin, I.T. 2002. Gene expression of 5-epi-aristolochene synthase and formation of capsidiol in roots of Nicotiana attenuata and N. sylvestris. Phytochemistry 60:109-116.

11 Bourgaud, F., Gravoy, A., Milesi, S., Gontier, E.2001. Production of plant secondary metabolites: a historical perspective. Plant Science 161: 839 - 851.

12 Briskin, D.P. 2000. Medicinal plants and phytomedicines. Linking plant biochemistry and physiology to human health. Plant Physiology 124:507-514.

13 Chaturvedi, D. 2011. Sesquiterpene lactones: structural diversity and their biological activities, In: Opportunity, Challenge and Scope of Natural Products in Medicinal Chemistry, Research Signpost, Kerala, India, Cap 10, 313-334pp.

14 Chen, H.C., Chou, C.K., Lee, S.D., Wang, J.C., Yeh, S.F. 1995. Active compounds from Saussurea lappa Clarks that suppress hepatitis B virus surface antigen gene expression in human hepatoma cells. Antiviral Research 27: 99-109.

15 Chen, Z., Gallie, D.R., 2008. Dehydroascorbate reductase affects non-photochemical quenching and photosynthetic performance. Journal of Biological Chemistry 283: 21347-21367.

16 Conforti F., Menichini F., Formisano C., Rigano D., Senatore F., Arnold N.A., Piozzi F. 2009. Comparative chemical composition, free radical-scavenging and cytotoxic properties of essential oils of six Stachys species from different regions of the Mediterranean Area. Food Chemistry 116: 898-905.

17 Demidchik V. 2015. Mechanisms of oxidative stress in plants: From classical chemistry to cell biology. Environmental and Experimental Botany 109:212-228.

18 Dewick, P. M. 2002. Medicinal natural products. A biosynthetic approach. New York, Wiley 507pp.

19 Foyer, C.H., Shigeoka, S. 2011. Understanding Oxidative Stress and Antioxidant Functions 
to Enhance Photosynthesis. Plant Physiology 155:93-100.

20 Galeotti, F., Barile, E., Curir, P., Dolci. M., Lanzotti, V. 2008. Flavonoids from carnation (Dianthus caryophyllus) and their antifungal activity. Phytochemistry Letters 1: $44-48$.

21 Gechev, T.S., Van Breusegem, F., Stone, J.M., Denev, I., Laloi, C. 2006. Reactive oxygen species as signals that modulate plant stress responses and programmed cell death. Bioessays 28: 1091-1101.

22 Hadacek, F. 2002. Secondary metabolites as plant traits: current assessment and future perspectives. Critical Reviews in Plant Sciences 21: 273-322.

23 Hansson, D., Menkis, A., Himmelstrand, K., Thelander, M., Olson, k, Stenlid J, Karlsson M, Broberg A. 2012. Sesquiterpenes from conifer root rot pathogen Heterobasidion occidentale. Phytochemistry 82: 158-165.

24 Harbone, J.B. 1999a. Classes and functions of secondary products, in: N.J. Walton, D.E. Brown (Eds), Chemicals from Plants: Perspectives on Plant Secondary Products. Londres, Imperial College Press, Cap 1, 1-26.

25 Harborne, J.B, Baxter, H., Moss, G.P. 1999b. Phytochemical dictionary: handbook of bioactive compounds from plants. Segunda edición, Londres: Taylor \& Francis.

26 Harborne, J. B. 2009. Introduction to ecological biochemistry, $4^{\text {th }}$ edition. Londres, Academic Press 380pp.

27 Hooper L., Cassidy, A. 2006. A review of the health care potential of bioactive compounds. Journal of the Science of Food and Agriculture. 86:1805-1813.

28 Inderjit, A., Weiner, J. 2001. Plant allelochemical interference or soil chemical ecology?. Perspective in PlantEcology Evolution and Systematics 4:4-12.

29 Jilani, G., Mahmood, S., Chaudhry, A.N., Hassan, I., Akram, M. 2008. Allelochemicals: sources, toxicity and microbial transformation in soil - a review. Annals of Microbiology 58: 351-357.

30 Jiménez J.P., Serrano, J., Tabernero, M., Arranz, S., Díaz, M.E., García, Goñi I., Saura-Calixto F. 2008. Effects of grape antioxidant dietary fiber in cardiovascular disease risk factors. Nutrition 24: 646-53.

31 Kaur, H., Kaur, R., Kaur, S., Baldwin, I.T., Inderjit. 2009. Taking ecological function seriously: soil microbial communities can obviate allelopathic effects of released metabolites. Plos One Journal 4: e4700.

32 Kerchev, P.I., Pellny, T.K., Vivancos, P.D., Kiddle, G., Hedden, P., Driscoll, S., Vanacker, H., Verrier, P., Hancock, R.D., Foyer, C.H. 2011. The transcription factor $\mathrm{ABI} 4$ is required for the ascorbic acid-dependent regulation of growth and regulation of jasmonate-dependent defense signaling pathways in Arabidopsis. Plant Cell 23: 3319-3334.

33 Kessler, A., Halischke, R., Baldwin, I. T. 2004. Silencing the jasmonate cascade: Induced plant defences and insect populations. Science 305: 665-668.

34 Kircher, H.W., Lieberman, F.V. 1967. Toxicity of tobacco smoke to the spotted alfalfa aphid Therioaphis maculata (Buckton). Nature 215: 97-98.

35 Kistler, H.C., Broz, K. 2015. Cellular compartmentalization of secondary metabolism. Frontiers in Microbiology 6:68.

36 Kossel, A. 1891. Über die Chemische Zusammensetzung der Zelle. Archiv für Physiologie 4:181 - 186 .

37 Kris-Etherton, P.M., Hecker, K. D., Bonanome, A., Coval, S.M., Binkoski, A.E., Hilpert, K.F, et al. 
2002. Bioactive compounds in foods: their role in the prevention of cardiovascular disease and cancer. American Journal of Medicine 113: 71-88.

38 Li, J., Ou-Lee, T.M., Raba, R., Amundson R. G., Last. R. L. 1993. Last Arabidopsis mutants are hypersensitive to UV-B radiation. The Plant Cell 5: 171-179.

39 Li, J.H., Liu, Y.O., Lü, P., Lin, H.F., Bai, Y., Wang, X.C., Chen, Y.L. 2009. A signaling pathway linking nitric oxide production to heterotrimeric $G$ protein and hydrogen peroxide regulates extracellular calmodulin induction of stomatal closure in Arabidopsis. Plant Physiology 150: 114-124.

40 Maldonado-Bonilla, L.D., Betancourt-Jiménez, M., Lozoya-Gloria, G. E. 2008. Local and systemic gene expression of sesquiterpene phytoalexin biosynthetic enzymes in plant leaves. European Journal of Plant Pathology 121: 439-449.

41 Martins, S. Mussatto, S. I., Martins, G., Saenz, J. M., Aguilar, C. N., Teixeira, J. A. 2011. Bioactive phenolic compounds: production and extraction by solid state fermentation. Biotechnology Advances 29: $365-373$.

42 Meng, J.C., Hu, Y.F., Chen, J.H., Tan, R.X. 2001. Antifungal sesquiterpenes and other constituents from Ajania fruticulosa. Phytochemistry 58: 1141-1145.

43 Minto, R.E., Blacklock, B.J. 2008. Biosynthesis and function of polyacetylenes and allied natural products. Progress in Lipid Research 47: 233-306.

44 Modzelewska, A., Sur, S., Kumar, S.K., Khan, S.R. 2005. Sesquiterpenes: natural products that decrease cancer growth. Current Medicinal Chemistry Anticancer Agents 5:477-499.

45 Nigam PS, Gupta N, Anthwal A. Pre-treatment of agro-industrial residues. 2009. In: Nigam PS, Pandey A, editors. Biotechnology for agro-industrial residues utilization. First ed. Netherlands: Springer, 13-33p.
46 Ogita, S., Uefuji, H., Yamaguchi, Y., Koizumi, N., Sano, H. 2003. RNA interference: Producing decaffeinated coffee plants. Nature 423: 823.

47 Pandey K. B., Rizvi S. I. 2009. Plant polyphenols as dietary antioxidants in human health and disease. Oxidative Medicine Cellular Longevity 2: 270-278.

48 Pandey D.K. 2009. Allelochemicals in Parthenium in response to biological activity and the environment. Indian Journal of Weed Science 41: 111-123.

49 Perry, L.G., Thelen, G.C., Ridenour, W.M., Callaway, R.M., Paschke, M.W., Vivanco, J.M. 2007. Concentrations of the allelochemical $( \pm)$-catechin in Centaurea maculosa soils. Journal of Chemical Ecology 33: 2337-2344.

50 Phung, T., Jung, H., Park, J.H., Kim, J.G., Back, K., Jung, S. 2011. Porphyrin biosynthesis control under water stress: sustained porphyrin status correlates with drought tolerance in transgenic rice. Plant Physiology 157:1746-1764.

51 Picman, A. 1983. Antifungal Activity of helenin and isohelenin. Biochemical Systematics and Ecology 11: 183-186.

52 Picman, A. 1986. Biological activities of sesquiterpene lactones. Biochemical Systematics and Ecology 14: 255-281.

53 Ralston, L., Tae kwon, S., Schoenbeck, M., Ralston, J., et al., 2001. Cloning, heterologous expression, and functional characterization of 5-epi-Aristolochene-1,3-Dihydroxylase from Tobacco (Nicotiana tabacum). Archives of Biochemistry and Biophysics 393: 222- 235.

54 Rosenthal, G. A., Berenbaum, M. R. 1992. Herbivores: their interactions with secondary plant metabolites. San Diego, Academic Press 452pp. 
55 Roze L.V., Chanda, A., Linz J.E. 2011. Compartmentalization and molecular traffic in secondary metabolism: a new understanding of established cellular processes. Fungal Genetics and Biology 48: 35-48.

56 Saad, M.M.G., Abdelgaleil, S.A.M., Suganuma, T. 2012. Herbicidal potencial of sesquiterpenes on wild oat, Avena fatia L. Biochemical Systematics and Ecology 44: 33 -337.

57 Seigler, D. S. 2003. Plant secondary metabolism. Boston, Dordrecht, London: Kluwer Academic Publishers 759pp.

58 Shoji, T., Yamada, Y., Hashimoto, T. 2000. Jasmonate induction of putrescine $\mathrm{N}$-methyltransferase genes in the root of Nicotiana sylvestris. Plant Cell Physiology 41: 831-839.

59 Stark, S., Kytöviita, M.M., Neumann, A.B. 2007. The phenolic compounds in Cladonia lichens are not antimicrobial in soils. Oecologia 152, 299-306.

60 Skaltsa H., Lazari D., Panagouleas C., Georgiadou E., Garcia B., Sokovic M. 2000. Sesquiterpene lactones from Centaurea thessala and Centaurea attica. Antifungal activity. Phytochemistry 55: 903-908.

61 Swain, T. 1977. Secondary compounds as protective agents. Annual Review of Plant Physiology 28:479-501.

62 van Breusegem, F., Dat, J.F. 2006. Reactive oxygen species in plant cell death. Plant Physiology 141: 384-390.

63 van Wyk, B. E., Wink, M. 2004. Medicinal plants of the world. Portland, USA, Timber Press Inc., 480pp.

64 Vialart, G., Hehn, A., Olry, A., Ito, K., Krieger, C., Larbat, R., Paris, C., Shimizu, B.-I., Sugimoto, Y., Mizutani, M., Bourgaud, F. 2012. A 2-oxoglutarate-dependent dioxygenase from Ruta graveolens L. exhibits p-coumaroyl CoA 2-hydroxylase activity ( $\mathrm{C} 2 \mathrm{H})$ : a missing step in the synthesis of umbelliferone in plants. The Plant Journal 70:460-470.

65 Voukeng I.K, Kuete V., Dzoyem J.P., Fankam A.G., Noumedem J.A.K., Kuiate J.R., Pages J.M. 2012. Antibacterial and antibiotic-potentiation activities of the methanol extract of some cameroonian spices against Gram-negative multi-drug resistant phenotypes. BMC Research Notes 5:299.

66 Wang, L. Y., Wei, K., Jiang, Y. W., Cheng, H., Zhou, J., He, W., Zhang C. C. 2011. Seasonal climate effects on flavanols and purine alkaloids of tea (Camellia sinensis L.). European Food Research and Technology 233: 1049-1055.

67 Wink, M. 2003. Evolution of secondary metabolites from an ecological and molecular phylogenetic perspective. Phytochemistry 64: 3-19.

68 Wink, M. 2006. Importance of plant secondary metabolites for protection against insects and microbial infections, Chapter 11. In: Rai, M., Carpinella, M.C. (Eds), Advances in Phytomedicine, Ed. Elsevier, 3:251-268.

69 Nuñez Y.O., Salabarria I.S., Collado I.G., Hernández-Galán R. 2007. Sesquiterpenes from the wood of Juniperus lucayana. Phytochemistry 68: $2409-2414$

70 Yazaki, K. 2004. Natural Products and Metabolites. In: Klee, H. and Christou, P., (Eds.) Handbook of Plant Biotechnology. John Wiley \& Sons Ltd. 811-857 pp.

71 Yen T.B., Chang H.T., Hsieh C.C., Chang S.T. 2008. Antifungal properties of ethanolic extracts and its active compounds from Calocedrus macrolepis var. formosana (Florin) wood. Bioresource Technology 99: 4871-4877. 